Article

\title{
Noise Pollution Prevention in Wind Turbines: Status and Recent Advances
}

\section{Ofelia Jianu *, Marc A. Rosen and Greg Naterer}

Faculty of Engineering and Applied Science, University of Ontario Institute of Technology, Oshawa, Ontario, L1H 7K4, Canada; E-Mails: Marc.Rosen@uoit.ca (M.A.R.); Greg.Naterer@uoit.ca (G.N.)

* Author to whom correspondence should be addressed; E-Mail: ofelia.jianu@uoit.ca; Tel.: +1-905-721-3268; Fax: +1-905-721-3370.

Received: 29 March 2012; in revised form: 17 April 2012 / Accepted: 9 May 2012/

Published: 29 May 2012

\begin{abstract}
The global push towards sustainability has led to increased interest in alternative power sources other than coal and fossil fuels. One of these sustainable sources is to harness energy from the wind through wind turbines. However, a significant hindrance preventing the widespread use of wind turbines is the noise they produce. This study reviews recent advances in the area of noise pollution from wind turbines. To date, there have been many different noise control studies. While there are many different sources of noise, the main one is aerodynamic noise. The largest contributor to aerodynamic noise comes from the trailing edge of wind turbine blades. The aim of this paper is to critically analyse and compare the different methods currently being implemented and investigated to reduce noise production from wind turbines, with a focus on the noise generated from the trailing edge.
\end{abstract}

Keywords: wind energy; wind turbine; noise reduction; aerodynamic noise

\section{Introduction}

Global warming and greenhouse gas emissions are of great concern. To reduce such emissions, there is a global trend towards cleaner energy sources. Promising alternatives for coal and other fossil fuels are nuclear power and renewable energy sources. One of the most promising renewable sources is wind energy. However, concerns exist with wind turbine technology, and one of the main ones is in 
the noise that occurs during operation. In order to successfully reduce or prevent the noise generated, the sources of noise must be identified. Two major sources of noise are present during operation: mechanical and aerodynamic. Mechanical noise generally originates from the many different components within the wind turbine, such as the generator, the hydraulic systems and the gearbox. Various mechanical noise prevention strategies exist, such as vibration suppression, vibration isolation and fault detection techniques, which will be described in this paper. Prevention strategies for aerodynamic noise are also discussed because it is the dominant source of noise from wind turbines. The largest contribution to aerodynamic noise comes from the trailing edge of wind turbine blades. Strategies for reducing aerodynamic noise include adaptive solutions and wind turbine blade modification methods. Adaptive noise reduction techniques include varying the speed of rotation of the blades and increasing the pitch angle. Although such strategies have been successfully implemented for noise reduction purposes, they can cause significant power loss. Therefore, alternative adaptive solutions are sought. Blade modification methods such as adding serrations have proven to be beneficial in reducing noise without any power loss.

This paper is organized as follows: noise sources are discussed in Section 2, noise reduction strategies are presented in Section 3 and conclusions are given in Section 4. Section 2 is subdivided into mechanical noise and aerodynamic noise. Section 3 is subdivided into mechanical noise reduction strategies, aerodynamic noise reduction strategies and use of exergy methods.

\section{Noise Sources}

Wind turbines have many components that generate noise. The noise disturbances by wind turbines are related to such factors as distance between the wind turbine and populated areas as well as the background noise where the wind turbine is operating [1]. Operating conditions and maintenance of the wind turbine also affect noise production [1]. In general, there are two main categories of noise sources for wind turbines: mechanical and aerodynamic. In this section, we briefly examine mechanical noise and its causes and solutions but aerodynamic noise is the main focus as it is considered the most significant form of noise and the most difficult to address. Figure 1 shows the different sources of noise and their respective sound power levels; a/b refers to airborne noise and $\mathrm{s} / \mathrm{b}$ refers to structural noise [2].

Figure 1. Horizontal axis wind turbine noise sources [2].

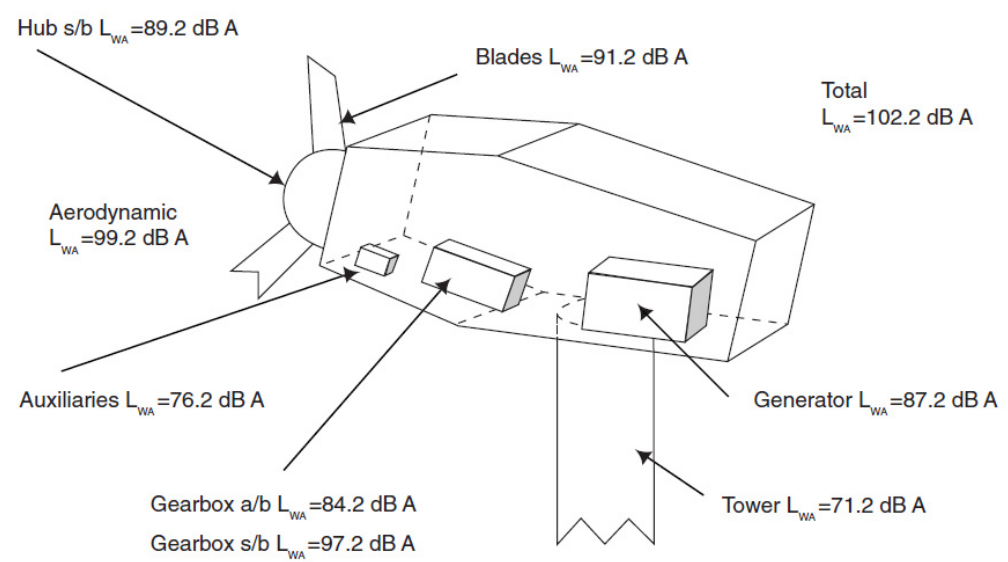




\subsection{Mechanical Noise}

Mechanical noise generally originates from the components within the wind turbine, such as the generator, the hydraulic systems and the gearbox. Other elements such as fans, inlets/outlets and ducts also contribute to mechanical noise. The type of noise produced by these mechanical components tends to be more tonal and narrowband in nature, which is more irritating for humans than broadband sound [1]. While the total wind turbine sound pressure level (SPL) only incurs a minor increase due to this noise, the penalty it places on wind turbines is much greater. Many countries have regulations which stipulate distances between wind turbines and the nearest buildings must be increased, or in some cases, outright refusal of installation, due to the negative impact of this noise on humans [1]. There are two ways in which mechanical noise is transmitted: airborne or structural. Airborne noise is straightforward, as the sound is directly emitted to the surroundings. Structural noise is more complex as it can be transmitted along the structure of the turbine and then into the surroundings through different surfaces, such as the casing, the nacelle cover, and the rotor blades [2]. The drive gearbox is a significant source of noise in wind turbines. The structure based noise generated by the tooth mesh propagates through the roller bearings of the gearbox and through the impact noise insulation to the nacelle bedplate and finally to the tower.

\subsection{Aerodynamic Noise}

Aerodynamic noise is more complex and, as can be seen from Figure 1 it is the dominant source of noise from wind turbines, with a sound power level of $99.2 \mathrm{~dB}$ A [2].

In general, there are six main regions along the blade (see Figure 2) [1-8]. These regions are considered to create independently their own specific noises, because the noises produced are fundamentally different and, since they occur in different regions along the blade, they do not interfere with each other [5]. The six regions are classified into turbulent boundary layer trailing edge noise, laminar boundary layer vortex shedding noise, separation stall noise, trailing edge bluntness vortex shedding noise, tip vortex formation noise and noise due to turbulent inflow.

Figure 2. Aerodynamic noise sources around a rotor blade due to wind flow with velocity, U [6].

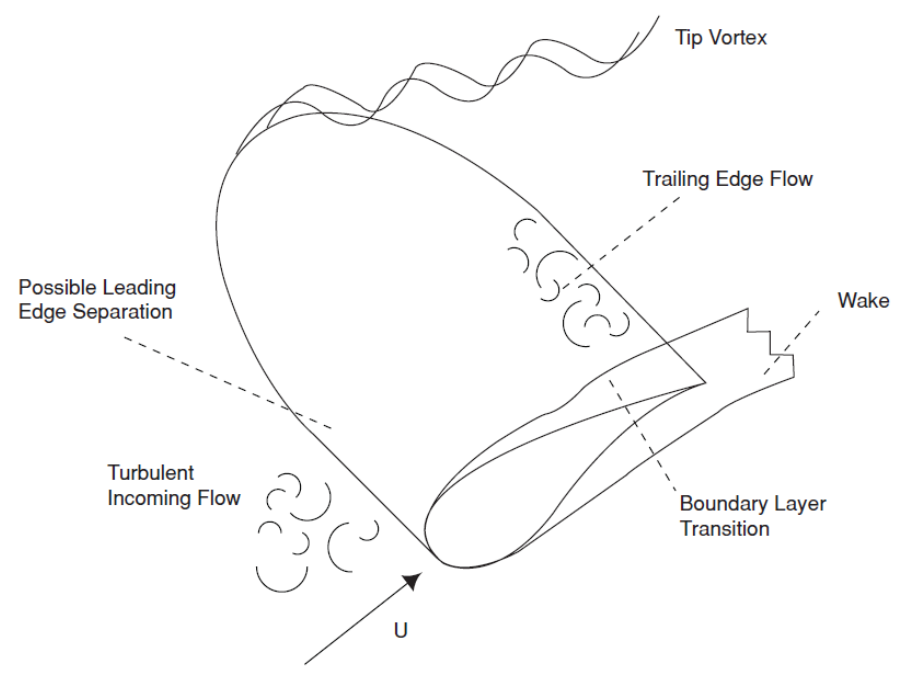


In general, there are six main regions along the blade (see Figure 2) [1-8]. These regions are considered to create independently their own specific noises, because the noises produced are fundamentally different and, since they occur in different regions along the blade, they do not interfere with each other [5]. The six regions are classified into turbulent boundary layer trailing edge noise, laminar boundary layer vortex shedding noise, separation stall noise, trailing edge bluntness vortex shedding noise, tip vortex formation noise and noise due to turbulent inflow.

\subsubsection{Turbulent Boundary Layer Trailing Edge Noise (TBL-TE)}

A predominant source of noise, turbulent boundary layer trailing edge noise (TBL-TE), results from the interaction of the boundary layer and the trailing edge of the airfoil, as depicted in Figure 3. Brooks and Hodgson [9] developed a predictor for TBL-TE using measured surface pressures. This predictor can be implemented provided sufficient information about the TBL convective surface pressure field passing the TE is available.

Figure 3. Turbulent boundary layer trailing edge noise [6].

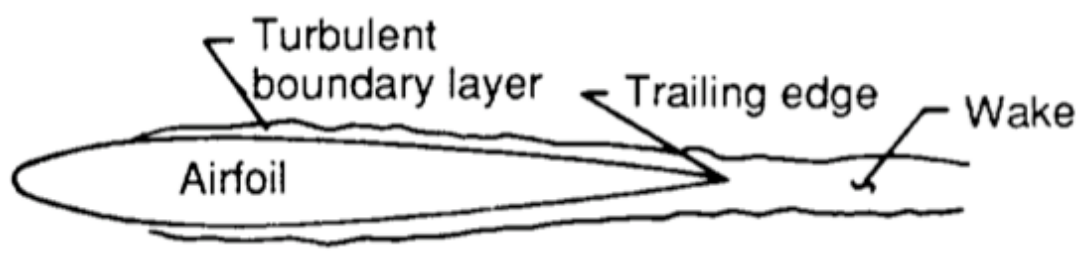

Schlinker and Amiet [10] employed a generalized empirical description of surface pressure to predict measured noise. An approach to model the turbulence within boundary layers as a sum of "hairpin" vortex elements was presented by Langley [6]. Ffowcs and Hall [11] present a simpler approach to the TBL-TE noise problem, based on an edge-scatter formulation. It has been found that the overall sound pressure level depends on velocity to the fifth power, through a number of studies [12]. Also, the Reynolds number and angle of attack have been shown to influence the turbulent structure [10,13]. Romero-Sanz and Matesanz [2] determined that TBL-TE noise can occur on both the suction and pressure sides of the airfoil and it is affected by the surface finish of the airfoil.

\subsubsection{Separation-Stall Noise}

Separation-stall noise occurs when the angle of attack increases from moderate to high as depicted in Figure 4. Since wind turbine airfoils operate at high angles of attack for significant portions of time, this source of noise is of significant interest. As the angle of attack increases, the boundary layer on the suction side increases and large-scale unsteady structures begin to form. It has been found that in such cases the noise increases by more than $10 \mathrm{~dB}$ relative to TBL-TE noise [4]. It has also been determined through far-field cross correlations that the noise is emitted from the trailing edge for mildly separated flow and from the chord for large-scale separation [13]. Empirical relations for separated-stall noise have been determined, and they are similar to those for TBL-TE noise with different scaling factors [2]. 
Figure 4. Separation-stall noise [6].
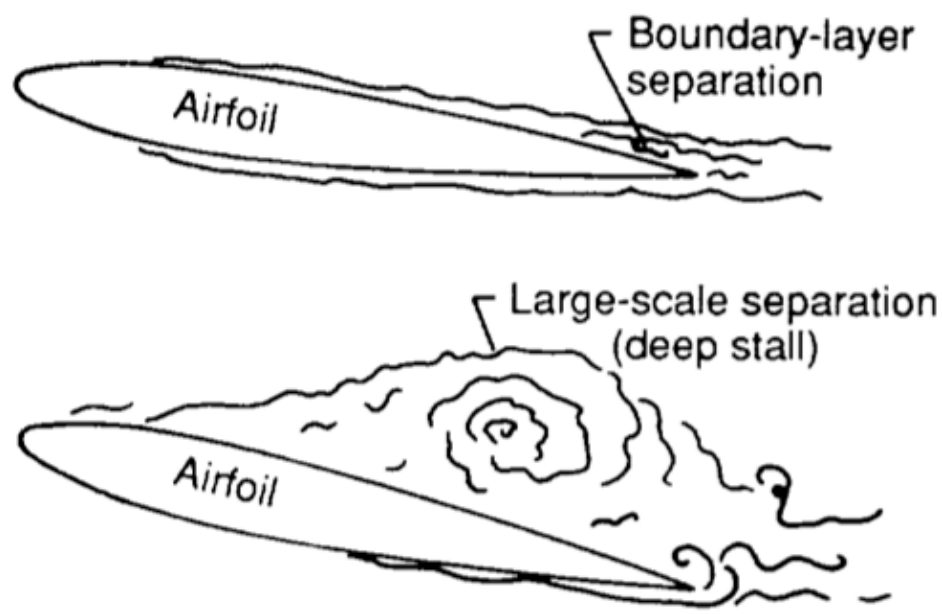

\subsubsection{Laminar-Boundary-Layer Vortex-Shedding (LBL VS) Noise}

This type of self-noise occurs when a laminar boundary layer is present over most or one side of the airfoil, as shown in Figure 5. The noise from this source is coupled to acoustically excited feedback loops taken between the trailing edge and instability waves (Tolmien-Schlichting waves) upstream of the trailing edge [6,7,14-19]. Pressure waves due to vortex shedding leaving the trailing edge propagate upstream and instabilities are amplified in the boundary layer. A feedback loop is created when the instabilities reach the trailing edge and vortices with similar frequency content form.

Figure 5. Laminar-boundary-layer vortex shedding noise [6].

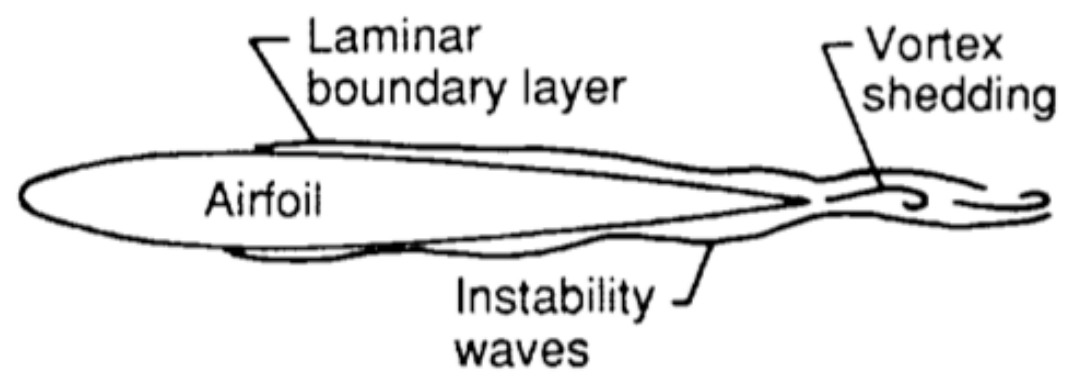

\subsubsection{Tip Vortex Formation Noise}

Unlike other noise sources due to its three dimensionality, this source of self-noise results from the interaction of the thick viscous turbulent core tip vortex with the trailing edge near the tip (see Figure 6) [6]. Experimental studies have isolated tip noise quantitatively [20]. A prediction model based on spectral data from delta wing studies, mean flow studies of several tip shapes and trailing edge noise analysis was proposed by George and Chou [13]. A relation for an untwisted, constant chord blade was also developed by Brooks, Pope and Marcolini $[6,12,20]$. 
Figure 6. Tip vortex formation noise [6].

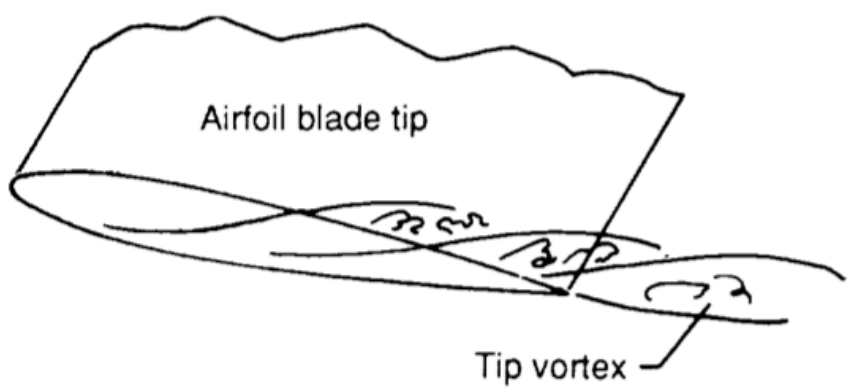

\subsubsection{Trailing-Edge-Bluntness Vortex-Shedding Noise}

Another important source of noise as determined by Brooks and Hodgson [9] is noise due to vortex shedding from blunt trailing edges, as represented in Figure 7. The frequency and amplitude of this noise source is given by the geometry of the trailing edge [2]. Empirical relations to predict this type of noise have been developed, and they are dependent on the trailing edge thickness and proportional to the sixth power of the velocity [2].

Figure 7. Trailing-edge-bluntness vortex-shedding noise [6].

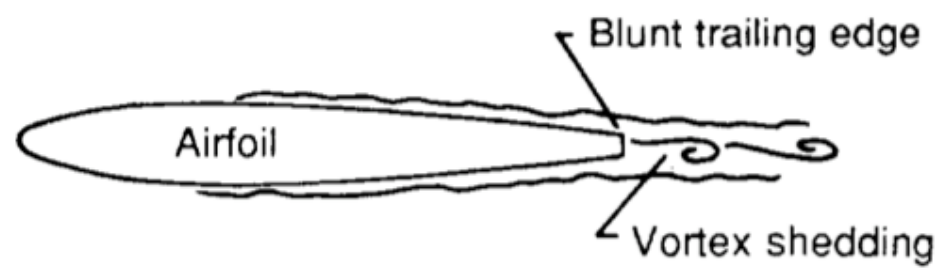

\subsubsection{Turbulent Inflow Noise}

At low frequencies, the interaction of the turbulent inflow with the leading edge of the turbine blades proves to be a significant source of noise. Figure 2 illustrates such sources of noise. Romero-Sanz [2] determined that depending on the size of the length scale relative to the leading edge radius of the airfoil, a dipole noise source (low frequency) or a quadrupole noise source (high frequency) could be created. Furthermore, the dipole noise source is dependent on the Mach number to the sixth power whereas the scattered quadrupole noise source is dependent on the Mach number to the fifth power [2]. Empirical relations have been developed by Lowson [21] for both low and high frequencies, based on the experimental results of Amiet [22].

\section{Noise Reduction Strategies}

There are many ways in which sound can be reduced. One is to design the wind turbine with acoustic behaviors in mind. Researchers are focused on reducing noise without affecting the power generated by the wind turbine. Strategies for reducing mechanical and aerodynamic noise will be discussed in this section. 


\subsection{Mechanical Noise Reduction}

One source of mechanical noise is vibrations induced by the rotating components. Vibration control is used to suppress or eliminate unwanted vibrations. Depending on the case, different control laws can be chosen in order to minimize unwanted vibration. Additionally, damping or increasing the effective mass can be realized by the controller [23]. Inferentially, the absorber works as an active system. This includes the use of sound isolating materials, insulation, and closing the holes in the nacelles which would decrease the sound transmitted to the air [6]. Aside from loss of power and increased maintenance costs, faulty gearboxes also increase noise levels in wind turbines. As a result, researchers are developing fault diagnostic systems for gearboxes with applications to wind turbines. A system has been developed to integrate singular value decomposition noise reduction, time-frequency analysis and order analysis methods in order to identify weak faults objectively and effectively [24]. More recently, efforts have been taken to develop intelligent techniques for online condition monitoring in machinery systems such as wind turbines. Several neural fuzzy classification techniques have been proposed for fault detection [25-29].

\subsection{Aerodynamic Noise Reduction}

\subsubsection{Adaptive Approaches}

There are a number of adaptive noise reduction approaches for aerodynamic noise, including varying the speed of rotation of the blades. Since an increase in rotational speed will also lead to increased noise production, lowering the rotational speed will lead to decreased sound. However the rotational speed decrease reduces power output, and therefore should only be implemented within a certain range of wind velocities, since high winds also have the added benefit of masking the sound of the wind turbine with the sound of the wind itself [2]. The pitch angle of the wind turbine blades also has an important role in noise production. An increase in pitch angle will lead to a reduction in the angle of attack. As the angle of attack increases, the size of the turbulent boundary layer on the suction side of the airfoil grows, thereby increasing noise production in the wind turbine. Therefore, if the pitch angle is increased, a thinner boundary layer results on the suction side, which is considered the strongest source of noise production [2]. This also implies that, on the pressure side, the effect is the opposite; therefore when using this method for noise control, it is important to find the appropriate pitch angle range for optimal noise control. As with the previous method, the major drawback to this adaptive noise control method is the corresponding reduction of power since the angle of attack is decreased. Despite a potential loss in power due to pitch angle changes that reduce noise, more wind turbines can potentially be built within a specified area [2].

\subsubsection{Wind Turbine Blade Modification Methods}

The main drawback to adaptive methods (an overall reduction of power) is a hindrance to that method of noise control. By breaking down the noise sources it can be seen that the maximum noise contribution occurs within the trailing edge [30,31]. Different aeroacoustic prediction models such as semi-empirical airfoil self-noise models and simplified theoretical models are available in the 
literature [32]. Kamruzzaman et al. [32] presented results using a well-known TNO-Blake model with Computational Aeroacoustics (CAA) and validated the predictions against wind tunnel measurements. The predictions are based on Reynolds Averaged Navier-Stokes simulation results. Such predictions are beneficial in determining the region with the highest aeroacoustic levels. Researchers established that the region between about 75-95\% span is exposed to the maximum flow velocities and it has the highest aero-acoustic noise levels [30]. Furthermore, experimental tests showed that most of the noise is generated when the blade is moving downwards in a clockwise rotation, as shown in Figure 8. Therefore, the majority of modification procedures are aimed at reducing the noise in this area. Research had been conducted into the use of aero-acoustically optimized airfoils and trailing edge serration [5], while trailing edge brushes have also been considered to control the noise generated from this portion of wind turbine blades [31].

Figure 8. Area on a turbine blade with largest sound production [2].

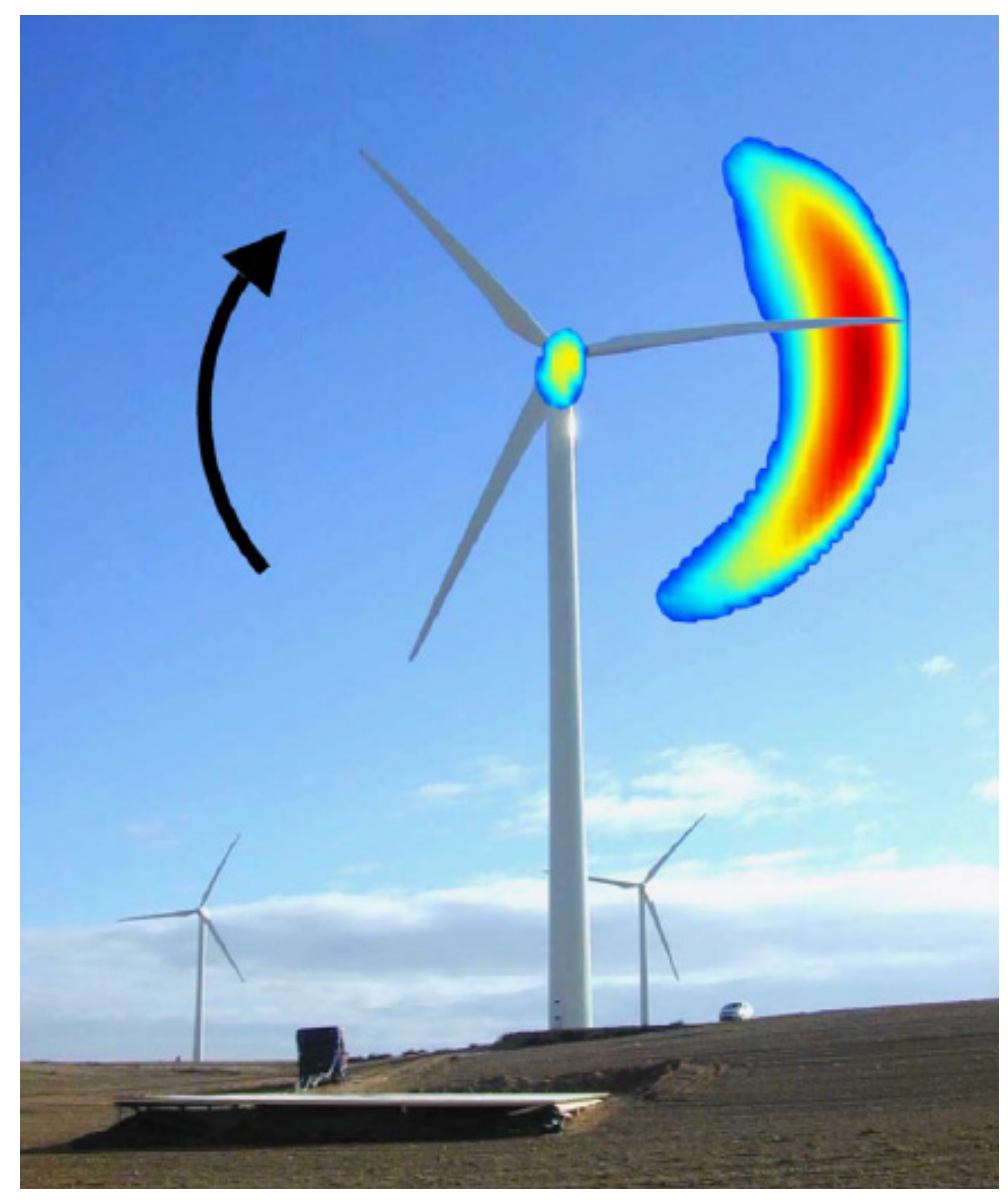

Many studies have focused on reducing noise from the trailing edge. One such method is through acoustically optimized airfoils. The "silent rotors by acoustic optimization" (SIROCCO) project was conducted in 2003-2007 in Europe [5], and involves having silent airfoils replace existing airfoils in the outer part of a baseline blade in the areas exposed to maximum flow velocities and highest aero-acoustic noise levels between $75-95 \%$ span. The airfoil design is shown in Figure 9. The SIROCCO project aimed to modify existing blades with a different trailing edge in the outer region of the blade, so designing an airfoil anew would lead to enhanced performance [5]. This project studied two different wind turbines: the Gamesa $850 \mathrm{~kW}$ turbine (G58) in Zaragoza, Spain with a rotor 
diameter of $58 \mathrm{~m}$, and the GE 2.3 MW turbine in Wieringermeer, Netherlands, with a rotor diameter of $94 \mathrm{~m}$. The acoustically optimized airfoils were initially tested in wind tunnels and then mounted on their respective turbines. Both turbines were tested using a hybrid rotor, and two baseline blades with one modified SIROCCO blade, which allowed for direct comparison of noise production with similar conditions (since all blades were on the same rotor).

Figure 9. Comparison between original and optimized airfoil [2].

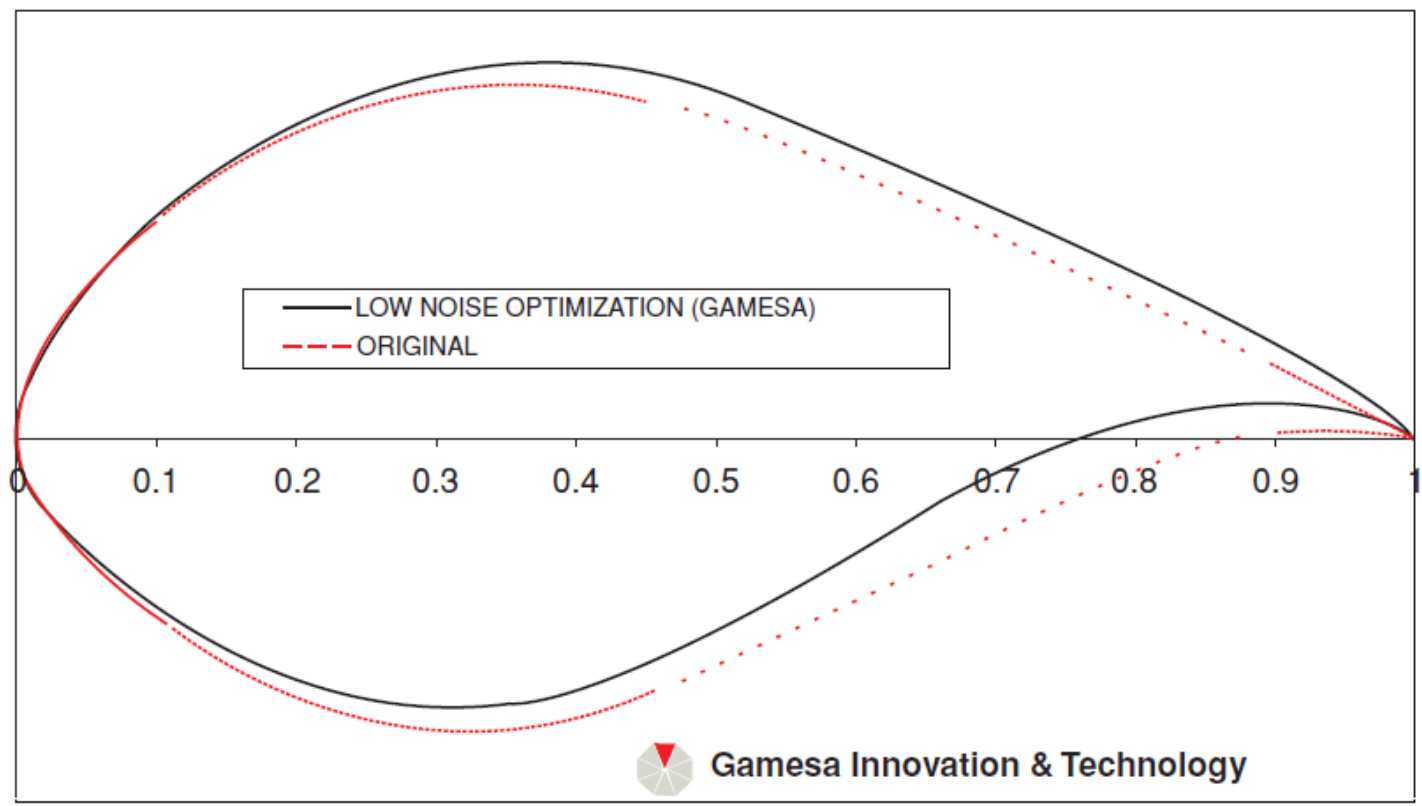

The noise reduction was found to be about 1-1.5 dB A for the G58 with a chord-based Reynolds number of $1.6 \times 10^{6}$ and 2-3 dB A for the GE94 with a chord-based Reynolds number of $3 \times 10^{6}$. It was difficult to analyze the aerodynamic performance differences since only 1 blade is different in the hybrid rotor. However, the power production of the hybrid rotor on the G54 with an average wind speed of $8 \mathrm{~m} / \mathrm{s}$ was reduced by $1.4 \%$, which was below the measurement uncertainty, but which the study attributed to an erosion trip used on the SIROCCO blade, and not due to the modification. For the GE94 turbine, the power production was higher than baseline; $2.8 \%$ annual energy production at an average wind speed of $8 \mathrm{~m} / \mathrm{s}$ [1].

In addition to having a SIROCCO blade, one of the blades on the GE turbine incorporated serrations on the baseline blades, as shown in Figure 10. The serrations were $2 \mathrm{~mm}$ thick, and mounted on the outer $12.5 \mathrm{~m}$ of the blade (on the pressure side). They were smoothed out using an epoxy filler to maintain the aerodynamics of the blade. The lengths of the serrations occurred from about $20 \%$ of the local chord, and varied as a function of radius. For example, they had a $10 \mathrm{~cm}$ tooth length at the tip and $30 \mathrm{~cm}$ at the most inboard position. The plane of serrations aligned with the flow direction at the blade trailing edge to maintain the aerodynamics of the blade. These serrations were found to reduce the noise by about $3.2 \mathrm{~dB}$ A at low frequencies. At higher frequencies, however, the noise production from the serrations is actually higher than the baseline blade [31]. The bluntness of the trailing edge also contributes to the overall noise production. The thickness of the trailing edge causes vortices which in turn generate sound. 
Figure 10. Trailing edge serrations [31].

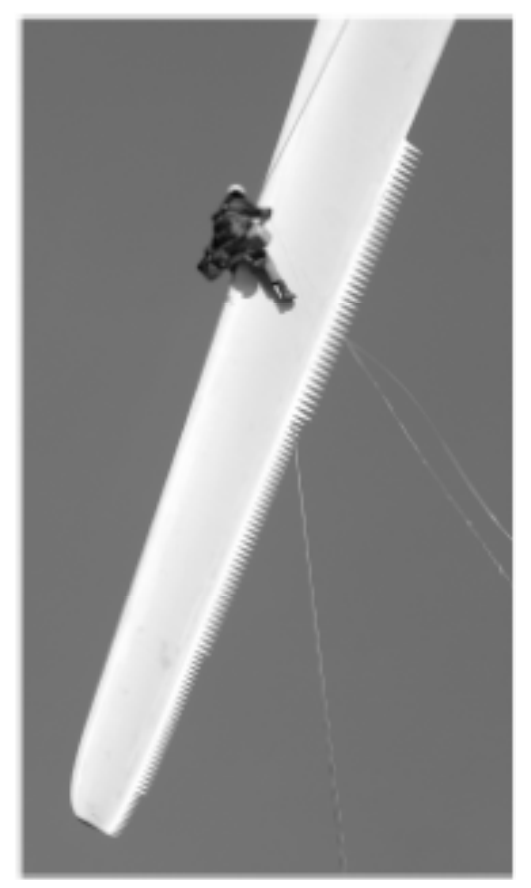

In order to minimize this effect, a single row of polypropylene fibres can be connected to the trailing edge, as shown in Figure 11. These brushes work similar to serrations, except that the tip angles in this case are extremely sharp. These brushes can reduce the sound by an amount depending on the brush design characteristics. Three fibre lengths $1_{\mathrm{f}}(15,30$ and $60 \mathrm{~mm})$ and fibre diameters $\mathrm{h}_{\mathrm{f}}(0.3,0.4,0.5 \mathrm{~mm})$ are considered [33]. These brushes were aligned automatically with trailing edge flow and showed significant noise reduction. Depending on the configuration and frequency, noise reductions from 2 to $10 \mathrm{~dB}$ were achieved. A possible problem to research with this modification is how well these brushes will perform in different weather conditions, such as snow and ice.

Figure 11. Trailing edge brushes [6].

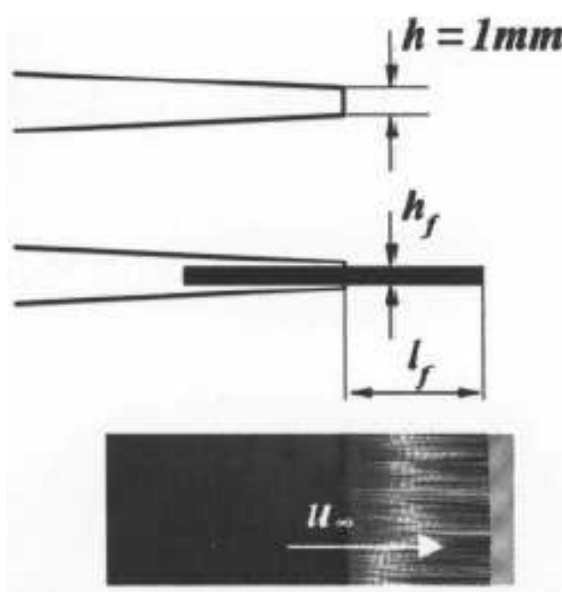

As shown in Figure 12, the serrations and optimized airfoils work very well at low frequencies, but in fact produce more noise at higher frequencies. At present, wind turbines contain controllers which control the angle of attack to optimize the power generation of the turbine [1]. Similar controllers 
could be used to change the blade between the different modes, such as using serrations at low frequencies, and then retracting them at higher frequencies to reduce the noise production at high frequencies. Furthermore, trailing edge brushes have been found to work well at high frequencies. In future designs, trailing edge brushes could be incorporated into blade design and, with a controller, could be retracted so they would only function within a specific frequency range.

Figure 12. Comparison of serrations vs. sirocco and baseline [30].

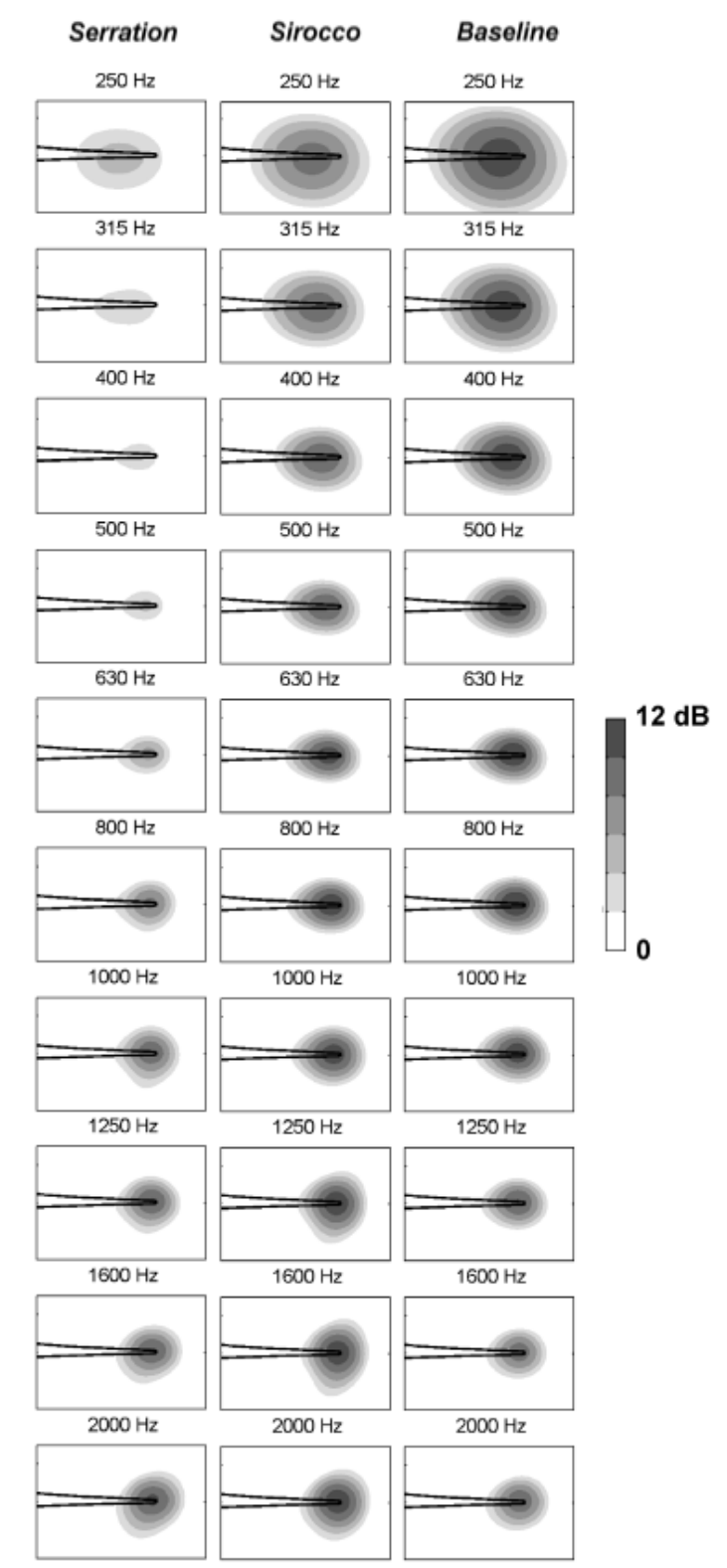

\subsection{Use of Exergy Methods}

An investigation has been undertaken by the authors to correlate the noise pollution to exergy, a factor not normally used to design wind turbine airfoils. The main objective was to determine a correlation between the sound pressure level resulting from flow over a solid object and the exergy destroyed within the fluid medium. This correlation has the potential to reveal relationships between 
noise pollution and exergy destruction. Such correlation may be useful in efforts to minimize noise pollution in commercial wind turbines.

\section{Conclusions}

Noise production in wind turbines and recent advances to prevent it have been critically reviewed and compared. To successfully reduce or prevent the noise generation, the sources of noise must be identified. Two major sources of noise are present during operation: mechanical and aerodynamic. Mechanical noise generally originates from the many different components within the wind turbine, such as the generator, the hydraulic systems and the gearbox. Different mechanical noise prevention strategies such as vibration suppression, vibration isolation and fault detection techniques are utilized for this type of noise. Aerodynamic noise is the dominant source of noise from wind turbines. It occurs at high velocities when turbulent boundary layers develop over the airfoil and they pass over the trailing edge or at lower velocities as a result of laminar boundary layers leading to vortex shedding at the trailing edge. Other factors presented include flow separation and blunt trailing edge flow leading to vortex shedding. Strategies for reducing aerodynamic noise include adaptive approaches and wind turbine blade modification methods.

\section{Acknowledgements}

The authors acknowledge the financial support from Natural Sciences and Engineering Council of Canada. The authors also acknowledge Mr. Ali Sherazee for his input.

\section{Conflict of Interest}

The authors declare no conflict of interest.

\section{References}

1. Klug, H. Noise from Wind Turbines: Standards and Noise Reduction Procedures. Paper presented on the Forum Acusticum, Sevilla, Spain, 16-20 September 2002.

2. Romero-Sanz, I.; Matesanz, A. Noise management on modern wind turbines. Wind Eng. 2008, $32,27-44$.

3. Oerlemans, S.; Sijtsma, P.; Mendez Lopez, B. Location and quantification of noise sources on a wind turbine. J. Sound Vib. 2007, 299, 869-883.

4. Moriarty, P.; Migliore, P. Semi-Empirical Aeroacoustic Noise Prediction Code for Wind Turbines; National Renewable Energy Laboratory: Golden, CO, USA, 2003.

5. Schepers, J.G.; Curvers, A.; Oerlemans, S.; Braun, K.; Lutz, T.; Herrig, A.; Wuerz, W.; Mantesanz, A.; Garcillan, L.; Fischer, M.; et al. SIROCCO: Silent Rotors by Acoustic Optimization. Presented at the Second International Meeting on Wind Turbine Noise, Lyon, France, 20-21 September 2007; ECN-M-07-064.

6. Brooks, T.F.; Pope, D.S.; Marcolini, M.A. Airfoil Self-Noise and Prediction; NASA Reference Publication 1218; National Aeronautics and Space Administration, Washinton, DC, USA, 1989. 
7. Leloudas, G.; Zhu, W.J.; Sorensen, J.N.; Shen, W.Z.; Hjort, S. Prediction and reduction of noise from 2.3 MW wind turbine. J. Phys. Conf. Ser. 2007, 75, doi:10.1088/1742-6596/75/1/012083.

8. Oerlemans, S.; Schepers, J.G. Prediction of wind turbine noise and validation against experiment. Int. J. Aeroacoustics 2009, 8, 555-584.

9. Brooks, T.F.; Hodgson, T.H. Trailing edge noise prediction from measured surface pressures. J. Sound Vib. 1981, 78, 69-117.

10. Schlinker, R.H.; Amiet, R.K. Helicopter Rotor Trailing Edge Noise; NASA CR-3470; NASA: Washington, DC, USA, 1981.

11. Ffowcs Williams, J.E.; Hall, L.H. Aerodynamic sound generation by turbulent flow in the vicinity of a scattering half plane. J. Fluid Mech. 1970, 40, 657-670.

12. Brooks, T.F.; Marcolini, M.A. Scaling of airfoil self-noise using measured flow parameters. AIAA J. 1985, 23, 207-213.

13. Chou, S.-T.; George, A.R. Effect of angle of attack on rotor trailing-edge noise. Am. Inst. Aeronautics Astronaut. J. 1984, 22, 1821-1823.

14. Paterson, R.W.; Amiet, R.K.; Munch, C.L. Isolated airfoil-tip vortex interaction noise. J. Aircraft 1975, 12, 34-40.

15. George, A.R.; Najjar, F.E.; Kim, Y.N. Noise Due to Tip Vortex Formation on Lifting Rotors. In Proceedings of the 6th Aeroacoustics Conference on American Institute of Aeronautics and Astronautics, Hartford, CT, USA, 4-6 June 1980.

16. Arakawa, C.; Fleig, O.; Iida, M.; Shimooka, M. Numerical approach for noise reduction of wind turbine blade tip with earth simulator. J. Earth Simul. 2005, 2, 11-33.

17. Tam, C.K.W. Discrete tones of isolated air-foils. J. Acoust. Soc. Am. 1974, 55, 1173-1177.

18. Fink, M.R. Fine structure of airfoil tone frequency. J. Acoust. Soc. Am. 1978, 63, doi:10.1121/1.2016551.

19. Wright, S.E. The acoustic spectrum of axial flow machines. J. Sound Vib. 1976, 45, 165-223.

20. Brooks, T.F.; Marcolini, M.A. Airfoil tip vortex formation noise. Am. Inst. Aeronautics Astronaut. J. 1986, 24, 246-252.

21. Lowson, M. Assessment and Prediction of Wind Turbine Noise; Flow Solutions Report 92/19, ETSU W/13/00284/REP; Energy Technology Support Unit: Harwell, UK, September 1992.

22. Amiet, R. Acoustic radiation from an airfoil in a turbulent stream. J. Sound Vib. 1975, 41, 407-420.

23. Kelly, S.G. Fundamentals of Mechanical Vibrations, 2nd ed.; McGraw Hill: New York, NY, USA, 2000.

24. Wang, F.; Zhang, L.; Zhang, B.; Zhang, Y.; He, L. Development of Wind Turbine Gearbox Data Analysis and Fault Diagnosis System. In Proceedings of the Power and Energy Engineering Conference (APPEEC), Whan, China, 25-28 March 2011.

25. Angelov, P.; Filev, D. An approach to online identification of Takagi-Sugeno fuzzy models. IEEE Trans. Syst. Man Cyber. Part B 2004, 34, 484-498.

26. Song, Q.; Kasabov, N. NFI-Neuro-fuzzy inference method for transductive reasoning and applications for prognostic systems. IEEE Trans. Fuzzy Syst. 2005, 13, 799-808.

27. Kasabov, N.; Song, Q. DENFIS: Dynamic, evolving neural-fuzzy inference systems and its application for time-series prediction. IEEE Trans. Fuzzy Syst. 2002, 10, 144-154. 
28. Wang, W.; Ismail, F.; Golnaraghi, F. A neuro-fuzzy approach for gear system monitoring. IEEE Trans. Fuzzy Syst. 2004, 12, 710-723.

29. Jianu, O. An Evolving Neural Fuzzy Classifier for Machinery Diagnostics. M.Sc. Thesis, Lakehead University, Thunder Bay, ON, Canada, 2010.

30. Oerlemans, S.; Schepers, J.G.; Guidati, G.; Wagner, S. Experimental Demonstration of Wind Turbine Noise Reduction Through Optimized Airfoil Shape and Trailing Edge Serrations. In Proceedings of the European Wind Energy Conference and Exhibition, Copenhagen, Denmark, 2-6 July 2001.

31. Oerlemans, S. Reduction of Wind Turbine Noise Using Optimized Airfoils and Trailing-Edge Serrations. In Proceedings of the 14th AIAA/CEAS Aeroacoustics Conference, Vancouver, BC, Canada, 5-7 May 2008.

32. Kamruzzaman, M.; Lutz, T.; Wurtz, W.; Shen, W.Z.; Zhu, W.J.; Hansen, M.O.L.; Bertagnolio, F.; Madsen, H.A. Validations and improvements of airfoil trailing-edge noise prediction models using detailed experimental data. Wind Energy 2012, 15, 45-61.

33. Herr, M. Experimental study on noise reduction through trailing edge brushes. New Results Numer. Exp. Fluid Mech. V 2006, 92, 365-372.

(C) 2012 by the authors; licensee MDPI, Basel, Switzerland. This article is an open access article distributed under the terms and conditions of the Creative Commons Attribution license (http://creativecommons.org/licenses/by/3.0/). 